\title{
Immunofluorescence in the diagnosis of herpes encephalitis
}

\author{
MAURICE LONGSON \\ Virology Laboratory, Department of Microbiology, United Manchester Hospitals, \\ Manchester M13 9WL
}

GARDNER (1970) has compared herpes encephalitis to smallpox; both present as virological emergencies which call for urgent answers. A suspected case of herpes encephalitis presents the virology laboratory with one of the rare occasions when it can reasonably be expected to provide a diagnosis within a few hours, at any time of the day or night. The emergence of the drugs discussed by Professor Lajhta today (1973) has led to attempts at 'blind' diagnosis by therapeutic trial, but I submit that this is both scientifically and ethically unjustified. The available drugs are poisons (Hardwick et al., 1969) and administration should be restricted to proved cases. Also, looking beyond the need of the individual patient, there is an urgent need to document the efficacy of the anti-herpes drugs at our disposal. Such an evaluation will only be significant if a diagnosis is achieved by the examination of patient material obtained before the institution of therapy.

Brain biopsy should not be undertaken lightly (Editorial, 1972) but it is probably a safe procedure and it provides the only method of obtaining a certain diagnosis of herpes encephalitis during life. The immunofluorescent examination of brain material for viruses is not the easiest of techniques and, in the hands of inexperienced workers, there have been many intractable problems. Many of the difficulties can now, however, be overcome and, if used with discrimination, the fluorescent antibody tracing of Herpesvirus $h$-simplex can provide a safe and ideal method for rapid diagnosis. Although not a substitute for virus culture, it will give an answer within 4 $\mathrm{hr}$ of anaesthesia of the patient.

Immunofluorescent diagnosis of herpes encephalitis was first reported by Sabin \& Messore (1961), using necropsy material, and by Sommerville (1966) using lymphocytes in lumbar fluid. The value of the last approach has never been confirmed and, in my experience, it is unreliable. The staining of virus antigen in brain biopsy tissue is a diagnostic technique which has been pioneered by our Chairman and his colleagues in Oxford (Tomlinson \& MacCallum, 1969). Indeed, for the purpose of this presentation, I will draw quite freely on the expertise of Dr A. H. Tomlinson from the Public Health Laboratory, Oxford, who has so successfully solved many of the technical problems involved (Tomlinson, 1972).

A needle biopsy specimen of infected brain is all that is required, although the unequal distribution of stainable antigen in different parts of the brain does mean that there is an element of luck in the operation. Careful pre-operative assessment of any focus of activity should help to load the dice in one's favour. The brain tissue should not be placed in formaldehyde, but can conveniently be immersed in virus transport solution, or in physiological saline. It is useful to have a clear strategy in mind; there is only a very small piece of material and many different types of investigation may be indicated. Because of the need to maintain sterility, the microbiologist should, in the first instance, be given custody of the whole specimen. He will apportion the tissue between himself and the various jars handed to him by electron microscopists, neuropathologists, biochemists and curators of liquid nitrogen refrigerators. Virus may be present in the grey or white matter, or in both, and it is sound practice to retain a fragment of each for both immunofluorescence and virus culture.

The pieces retained for immunofluorescence may be used to prepare impression smears or, much more preferably, they may be mounted on the cryostat chuck and quenched in isopentane or liquid nitrogen for the preparation of 3-5 $\mu \mathrm{m}$ sections. The slide preparations are rapidly air-dried and fixed for $\mathbf{1 0}$ min in anhydrous acetone, after which they are either stained, or they may be stored for many weeks at $-20^{\circ} \mathrm{C}$. If necessary, acetone-fixed slides may be sent away through the post.

Immunofluorescent staining of the brain preparation can be accomplished by an indirect technique, but I would very much recommend the direct approach, with a single conjugated anti-herpes serum. In my experience, this method is very much the safer. The serum is prepared in young rabbits and, in an effort to minimize the formation of non-specific antibodies, the immunizing antigen is grown in rabbittissue culture. The animals are intensely immunized, but only a minority of the sera are likely to be usable; most rabbits, for reasons which are entirely obscure, yield sera which, though potently anti-herpetic, 
produce a totally unacceptable degree of non-specific staining of tissue and cells. I cannot recommend any particular immunizing schedule or any particular breed of rabbit - the whole procedure appears to be entirely empical, one serum is satisfactory and the next half-dozen are hopeless. It must be stressed that careful selection of the final reagent used for routine tests is mandatory. I am not aware of any commercially available material.

There are many acceptable methods of coupling antibody and fluorescein, but perhaps the method described by Clark and Sheppard (1963) presents the most satisfactory approach. In any event, it is important not to add too much fluorescein by keeping the dye-protein ratio down to $1: 40$, or less. Careful attention to the preliminary fractionation of the serum and to the details of the conjugation procedure, should make it unnecessary to further purify the conjugate by DEAE cellulose chromatography, although absorption against liver powder or tissue culture cells may be helpful. It should be possible with a good serum to use the reagent at a $1: 20$ or $1: 60$ dilution. With a $1: 40$ dye-protein ratio, this dilution will mean that the virus antigen fluorescence may be weak, but this is the price of of specificity. I am sorry to have to keep returning to this problem of specificity, but it deserves emphasis. It is one thing to stain specially prepared research tissue culture preparations, and it is quite another to look at a biopsy specimen and to have to give a verdict which may well affect a patient's chance of survival. When one has a patient at death's door and a team of neurologists and neurosurgeons pacing outside it is most uncomfortable not to have absolute confidence in one's reagent and to be worried about the degree of 'greenness' of perhaps no more than five positive cells in a smear.

Whereas it is true that too much emphasis can be placed on problems of instrumentation, I do believe that a suitably equipped microscope can make a big difference to the immunofluorescent examination of brain tissue. Fortunately, the ideal microscope is not sophisticated and can be relatively cheap. The critical specifications include:

(1) Objectives-moderately low-powered such as a 20 magnification. This will allow ready scanning of large areas of the preparation and will ensure adequate brightness of the image, even with low concentrations of dye. Image-brightness can be improved by the use of an objective with a high numerical aperture, and systems such as the Wild 'Fluotar' 20 NA 0.65 or the Vickers Apochromat 20 NA 0.65 can be recommended.

(2) Condensers - a good quality and efficient cardiod darkground. Brightfield condensers are not satisfactory for immunofluorescent tracing in circumstances where contrast is important whereas incident-light excitation does not confer any advantage in the diagnostic test we are considering. Quartz glass is unnecessary and dry darkground condensers are unsuitable. Image brightness can be improved by the use of a toric lens such as is incorporated in the Tiyoda and Reichert systems. This is not, however, indispensable, but an attempt should be made to match the size of the filament image to the lower aperture of the darkground condenser.

(3) Light source-a $100 \mathrm{~W}$ (or $150 \mathrm{~W}$ ) quartzhalogen lamp together with a correctly designed housing and collector lens. Fluorescein-protein complexes are maximally excited by blue light at $495 \mathrm{~nm}$ (Plate 1) and it is unnecessary, expensive and confusing to rely on the relatively less efficient excitation possible with ultra-violet radiation. When brain tissue is exposed to u.v. excitation, it autofluoresces. This emission is predominantly blue, but there are significant yellow and green components. The blue fluorescence may be dazzling and, if so, it will quench any pale specific immunofluorescence. A yellow secondary filter will remove the blue emission but any refractile material in the brain will now appear green and may cause serious confusion (Plate 2).

(4) Filters-an interference filter of the so-called 'F.I.T.C.' type, a Schott OG $5152 \mathrm{~mm}$ thick secondary filter and a selection of 1,2 and $3 \mathrm{~mm}$ thick red cutting Schott BG 38 filters (Plate 3). Suitable interference filters are manufactured by Balzer's in Liechtenstein, and by Optisk Laboratories in Denmark. Plate 4 depicts, in purple, the transmission curve of such a filter and three salient features can be noted; (a) there is a $70-80 \%$ transmission of blue light at a point corresponding to the excitation peak of fluorescein at $495 \mathrm{~nm}$; (b) there is no transmission in the green-yellow bands above $510 \mathrm{~nm}$, and hence, the exciting cone of light reaching the object will contain no wavelengths which could be confused with the fluorescent emission of fluorescein at $525 \mathrm{~nm}$ (Plate 1), and (c) there is a substantial transmission of red light in the $700 \mathrm{~nm}$ region. Plate 4 also shows (in yellow) the transmission curve of an OG 515 secondary filter: this is placed between the object and the observer and if correctly selected, will occlude all the exciting wavelengths, but will transmit as much as possible of the fluorescein $525 \mathrm{~nm}$ emission. It will be appreciated that between $495 \mathrm{~nm}$ and $525 \mathrm{~nm}$ there is little room for manoeuvre but that any failure in the performance of either primary or secondary filter will cause a series of difficulties which could be cumulative. There may easily be a loss of excitation energy and hence of image brightness, or there may be transmission of green light all the way from the light source to the microscopist, or the secondary filter may seriously attenuate the light emitted by the fluorescein. Particular care must be 


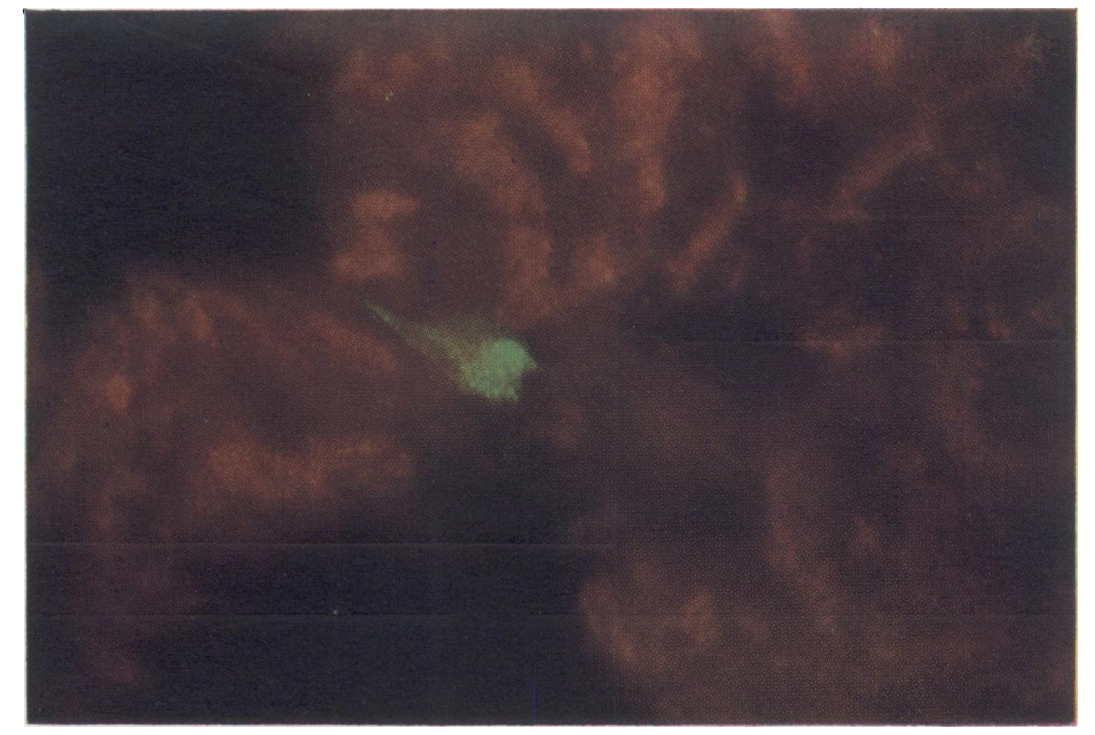

Plate 6. Neurone in brain biopsy from a case of herpes encephalitis (Liversedge, 1973) showing positive fluorescence. (See text page 405.)

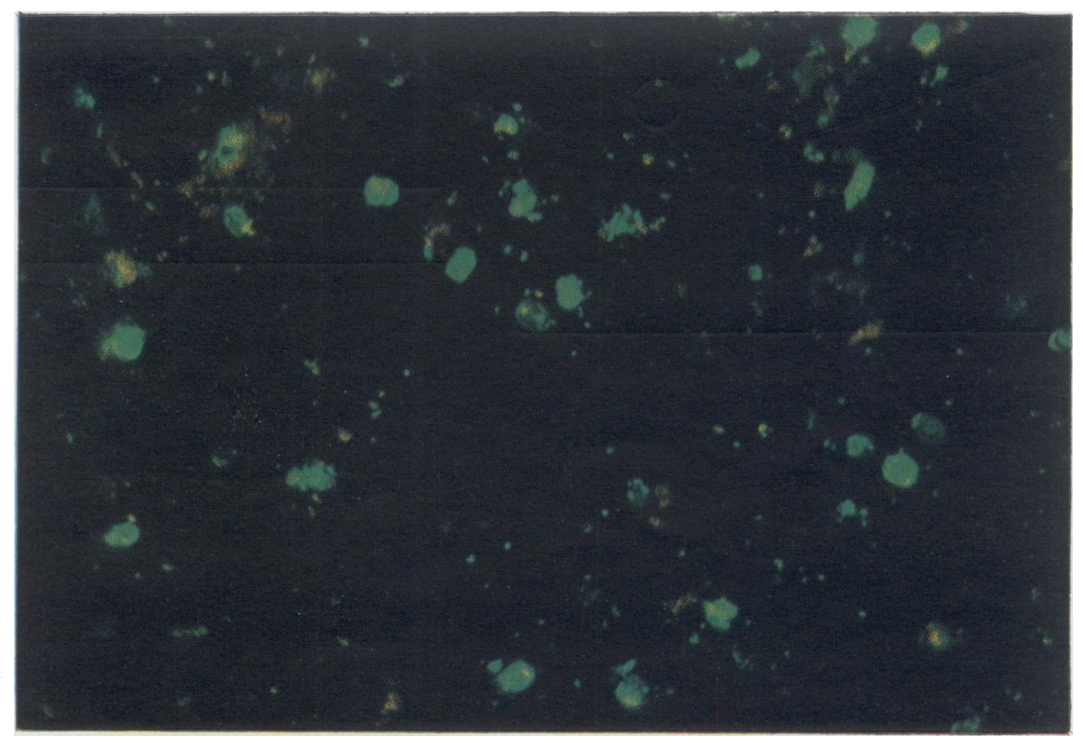

Plate 1 (of Discussion commencing on p. 406). Immunofluorescent staining of herpes simplex virus of infected cells in a frozen section of brain biopsy. Control preparation showed no green fluorescence. (See text page 408.) 

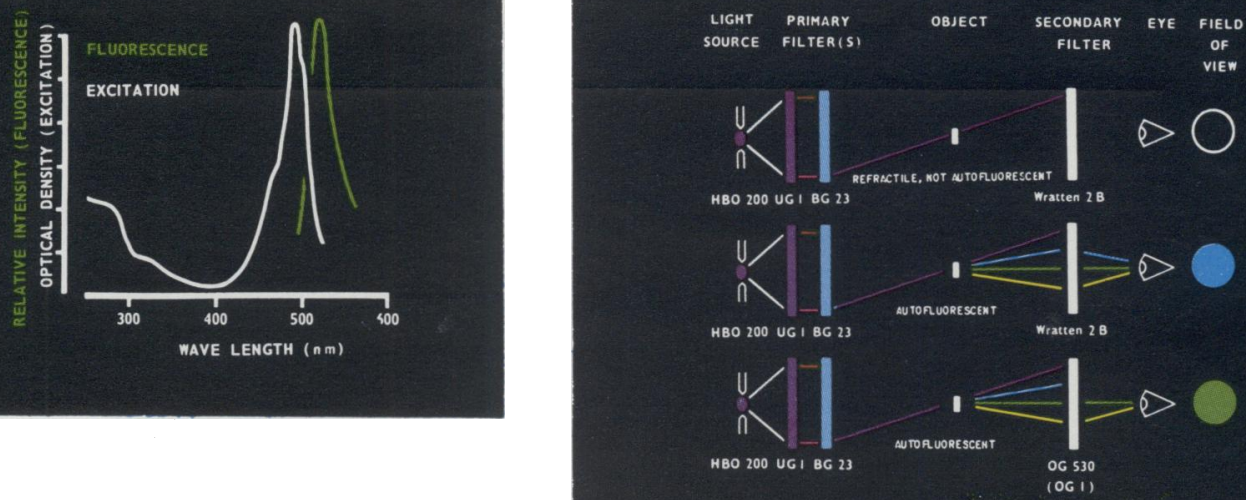

DARK FIELD ULTRA-VIOLET LIGHT FLUORESCENT MICROSCOPY

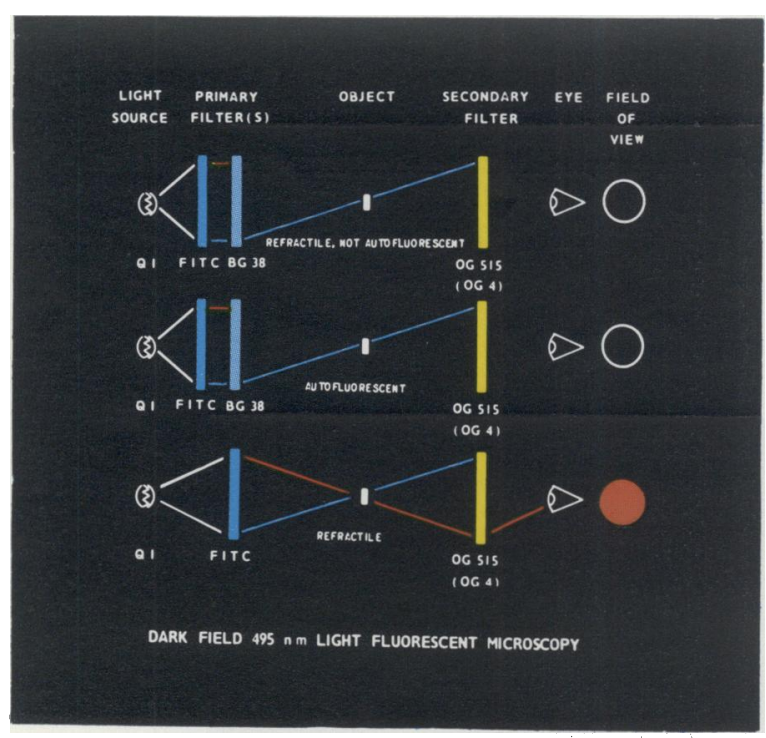

\section{Plate 3}

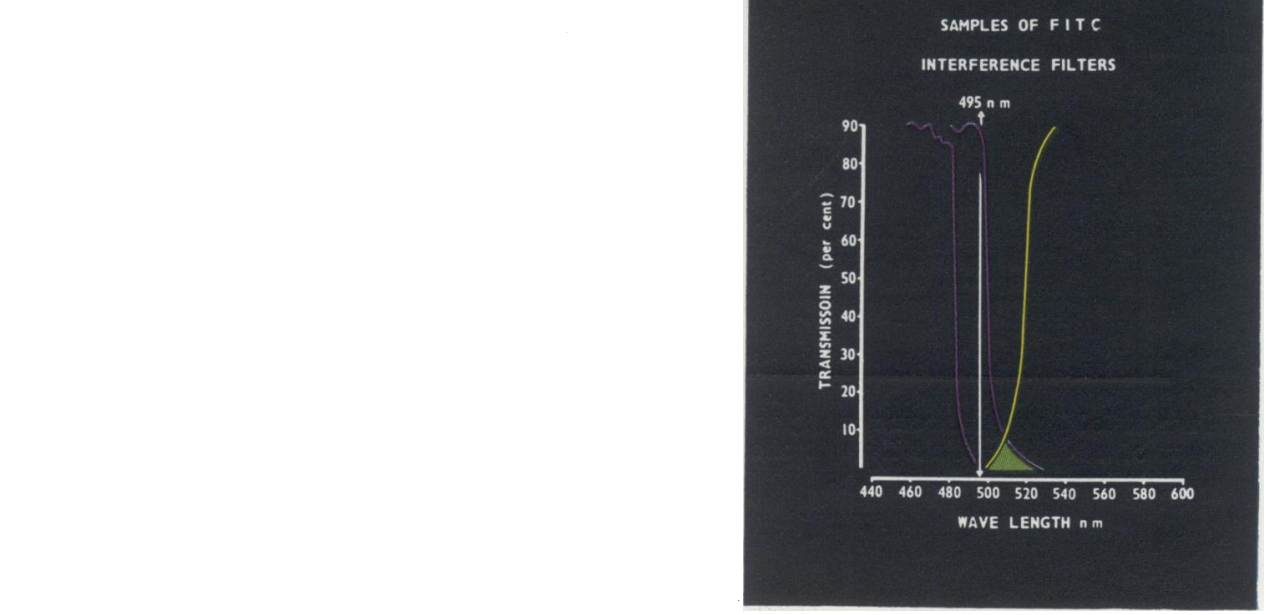

Plate 5

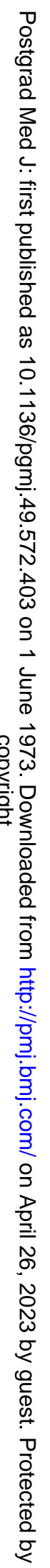


given to the choice of the interference filter. Plate 5 depicts the results obtained with two such filters, each bearing the same manufacturer's identical code number. The filter on the left transmitted little or no light at $495 \mathrm{~nm}$ and the intensity of specific fluorescence was, not surprisingly, seriously affected. The other primary filter transmitted maximally at $495 \mathrm{~nm}$, but also transmitted a significant amount of green light at $510-520 \mathrm{~nm}$, which was of course transmitted to the microscopist by the secondary yellow filter. The red transmission through the primary filter is not a cause of confusion. Indeed, refractile material in the object, such as brain myelin, will appear red to the observer and will form a useful contrast against the green specific fluorescence of herpetic neurones (Plate 6). Any intense or otherwise objectionable red background can be attenuated or eliminated by the addition of varying thicknesses of BG 38 glass (Plate 3).

I believe that it is only by careful attention to detail that viral immunofluorescence will become accepted as a safe diagnostic procedure, but that the test will increasingly prove itself to be of value in uncovering the many cases of herpes encephalitis which now remain undiagnosed.

\section{Acknowledgments}

I wish to thank Dr A. H. Tomlinson for many patient hours of advice and $\mathrm{Mr}$ Andrew Bailey for enthusiastic technical assistance. Most of the work which forms the basis of this presentation was performed in the Department of Bacteriology and Virology, University of Manchester.

\section{References}

Clark, H. F. \& Sheppard, C. C. (1963) A dialysis technique for preparing fluorescent antibody. Virology, 20, 642.

Editorial (1972) Herpes encephalitis. British Medical Journal, 1, 582.

Gardner, P. S. (1970) Rapid diagnostic techniques in clinical virology. In: Modern Trends in Medical Virology (Ed. by R. B. Heath and A. P. Waterson), vol. 2, p. 15. Butterworth, London.

Hardwick, J. L., Lajhta, L. G., Beswick T. S. L. \& LongSON, M. (1969) Treatment of herpetic stomatitis with idoxuridine. British Dental Journal, 126, 247.

LAJHTA, L. G. (1973) Molecular pharmacology of the pyrimidine analogues. Postgraduate Medical Journal, 49, 410.

Liversedge, L. A. (1973) Clinical features of herpes simplex encephalitis (acute necrotizing encephalitis). Postgraduate Medical Journal, 49, 383.

Tomlinson, A. H. (1972) Tungsten halogen lamps and interference filters for immunofluorescent microscopy. Proceedings of the Royal Microscopial Society, 7, 1.

Tomlinson, A. H. \& MacCallum, F. O. (1969) Virological diagnosis of herpes simplex encephalitis. In: Virus Diseases and the Nervous System (Ed. by C. W. M. Whitty, J. T. Hughes and F. O. MacCallum). Blackwell Scientific Publications, Oxford.

Sabin, A. B. \& Messore, G. (1961) A fluorescent antibody technique in the study of fixed tissues from patients with encephalitis. In: Encephalitides (Ed. by L. Van Bogaert et al.), p. 621. Elsevier, Amsterdam.

Sommerville, R. G. (1966) Rapid identification of neurotropic virus by an immunofluorescent technique applied to cerebrospinal fluid cellular deposit. Archives gesamte Virus Forschung, 19, 63.

Plate 1. Optical density (absorption) and intensity of fluorescence of a fluorescein isothiocyanate-globulin conjugate.

Plate 2. Diagrammatic representation of ultra-violet light fluorescence microscopy of an object which does not contain any fluorescent dye. The diagrams show the selective effect of the u.v. passing-visible/light-cutting primary filters and the effect on the microscope field of an autofluorescent object with either a colourless or a yellow secondary filter.

Plate 3. Diagrammatic representation of blue light $(495 \mathrm{~nm})$ fluorescence microscopy of an object which does not contain fluorescent dye, using a quartz-halogen light source. The diagrams show the absence of autofluorescence and the effect on the field of a red cutting filter.

Plate 4. Transmission curves of F.I.T.C. interference and yellow secondary filters. Note small area of overlap (green triangle).

Plate 5. Transmission curves of rejected F.I.T.C. interference filters. Note magnitude of overlap between primary and secondary filters and contrast with Plate 4. 